\title{
Namen von phantastischen Wesen und Orten in den Türkischübersetzungen von Michael Endes Fantasyroman Die unendliche Geschichte
}

\section{Names of Fantastic Beings and Places in the Turkish Translations of Michael Ende's Fantasy Novel Die unendliche Geschichte}

\section{Michael Ende'nin Die unendliche Geschichte Adlı Romanının Türkçe Çevirilerindeki Fantastik Varlık ve Yerlerin İsimleri}

\author{
Gülsüm Uğuz ${ }^{1}$, Z, Zehra Gülmüş² (i)
}

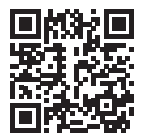

'Anadolu University, School of Foreign Languages, Eskişehir, Turkey

${ }^{2}$ Anadolu University, Faculty of Letters, Department of Translation and Interpreting, Eskişehir, Turkey

ORCID: G.U. 0000-0002-6615-1835; Z.G. 0000-0001-8040-693X

Corresponding author: Gülsüm Uğuz (Lecturer), Anadolu University, School of Foreign Languages, Eskişehir, Turkey E-mail:gulsumuguz@anadolu.edu.tr

\section{Submitted: 15.09.2019}

Accepted: 20.06 .2020

Citation: Uguz, G., \& Gulmus, Z. (2020). Names of Fantastic Beings and Places in the Turkish Translations of Michael Ende's Fantasy Novel Die unendliche Geschichte. Istanbul Üniversitesi Çeviribilim Dergisi - Istanbul University Journal of Translation Studies 12, (2020), 1-16. https://doi.org/10.26650/iujts.2020.12.0001

\section{ZUSAMMENFASSUNG}

Die Übersetzung der Kinder- und Jugendliteratur nimmt in der Translationswissenschaft eine besondere Stellung ein, da sie mit zahlreichen Übersetzungsproblemen verbunden ist. Dennoch liegen hierzu bislang nur wenige translationswissenschaftliche Untersuchungen vor. Mit der vorliegenden übersetzungskritischen Analyse von Michael Endes Fantasyroman Die unendliche Geschichte (1979) und dessen Übersetzungen ins Türkische soll ein Beitrag zur Schließung dieser Forschungslücke geleistet werden. Es liegen insgesamt vier Türkischübersetzungen vor, vorbereitet von zwei Übersetzern: Saadet Özkal (Ende 1986; 1999; 2017) und Saffet Günersel (Ende 1996). Alle vier Übersetzungen tragen denselben Titel Bitmeyecek Öykü. Die Übersetzer werden aufgrund von zahlreichen visuellen, sprachlichen und stilistischen Merkmalen des Kinder- und Jugendromans vor verschiedenen Übersetzungsproblemen gestellt. Hierzu gehören u. a. die Namen von phantastischen Wesen und Orten, die in der Romanhandlung eine wichtige Rolle spielen und die literarische Kreativität des Autors zum Vorschein bringen. Die Analyse von repräsentativen Namen anhand eines funktionalistisch und kindeszentrisch übersetzungskritischen Ansatzes (C. Nord und E. O'Sullivan) zeigt, dass die von den Übersetzern zur Lösung dieses Problems bevorzugten Übersetzungsverfahren zu recht unterschiedlichen Übersetzungen führen. Anhand der Beispielanalysen wird deutlich, dass Özkals Übersetzungen eher ausgangstextorientiert sind und Günersels Übersetzung eher funktional orientiert und somit mehr rezipientengerichtet ist.

Schlüsselwörter: Kinder und Jugendliteratur, Die unendliche Geschichte, Bitmeyecek Öykü, Funktionale Übersetzungskritik, Übersetzungsverfahren

\section{ABSTRACT}

The translation of children's and youth literature is of particular importance in Translation Studies but also poses many translation problems. However, there 
has so far been limited research that focuses on translation criticism in the field of children's and youth literature. The aim of this study is to contribute to discussions on the translation of children's and youth literature based on the example of Michael Ende's fantasy novel Die unendliche Geschichte (1979) and its Turkish translations. Overall, there are four Turkish translations done by two translators Saadet Özkal (Ende 1986; 1999; 2017) and Saffet Günersel (Ende 1996). All translations have the same Turkish title Bitmeyecek Öykü.Translation work on the children's fantasy novel Die unendliche Geschichte involves many potential translation problems not least due to the use in the novel of specific visual, linguistic and stylistic means. Specific translation challenges in the novel include, inter alia, those that arise due to the prevalence of names of important fantastic beings and places, which indicate the author's creativity. This analysis, conducted within the scope of functional and child-centered theory of translation (C. Nord and E. O'Sullivan), shows that different translation techniques were used by the translators to overcome the particular translation challenges in the novel in question. The results suggest that Özkal's translations are source text oriented, and Günersel's translation is rather functional and target reader oriented.

Keywords: Michael Ende, Die unendliche Geschichte, Bitmeyecek Öykü, Functional Translation Criticism, Translation Techniques

\section{Öz}

Çocuk ve gençlik edebiyatı ürünleri çevirisinin çeviribilim çalışmaları alanında özel bir konuma sahip olmasına ve beraberinde birçok çeviri sorunu getirmesine karşın bu alanda yapılan çeviri eleştirisi incelemeleri sınırlı sayıdadır. Çalışmanın amacı, Alman yazar Michael Ende'nin Die unendliche Geschichte (1979) adlı ünlü fantastik çocuk ve gençlik romanının Türkçe çevirileri örneğinde çocuk ve gençlik edebiyatı çevirisi alanına katkı sağlamaktır. Eserin toplamda Saadet Özkal (Ende 1986; 1999; 2017) ve Saffet Günersel (Ende 1996) tarafından hazırlanan dört çevirisi bulunmaktadır. Tüm çeviri eserler Bitmeyecek Öykü ismiyle yayımlanmıştır. Çevirmenlerin birçok çeviri sorunu ile karşılaşması, Die unendliche Geschichte adlı çocuk fantastik romanının çok sayıda kendine özgü görsel, dilsel ve biçemsel öğeler içermesinden kaynaklanmaktadır. Eserde bir çeviri sorunu oluşturan unsurlardan bir tanesi eserde büyük bir önem taşıyan ve yazarın yaratıcılığını ön plana çıkaran fantastik varlık ve yerlerin isimleridir. Çalışmada işlevsel ve çocuk/ genç okur odaklı çeviri kuramı ısığında (C. Nord ve E. O'Sullivan) çeviri sorunu oluşturan fantastik varlık ve yer isimleri örnekleri ele alınmış, çevirmenlerin söz konusu çeviri sorunlarını aşmak için kullandıkları çeviri tekniklerinin farklılık gösterdiği saptanmıştır. Örnek incelemelerin sonuncunda Özkal'ın çeviride daha çok kaynak metin odaklı, Günersel'in ise daha çok çevirinin işlevini dikkate alarak erek okur odaklı bir yaklaşımı benimsediği görülmektedir

Anahtar kelimeler: Michael Ende, Die unendliche Geschichte, Bitmeyecek Öykü, İ̧levsel Çeviri Eleştirisi, Çeviri Teknikleri 


\section{Einleitung}

Die Kinder- und Jugendliteratur (KJL) ${ }^{1}$ wurde in der Translationswissenschaft lange Jahre vernachlässigt und als Sonderfall betrachtet (vgl. hierzu z. B. Reiss 1982: 7; O'Sullivan 1991/1992: 4; Koller 2011: 203). Grund dafür ist die auch heute noch oft anzutreffende Vorstellung, dass das Übersetzen von KJL im Vergleich zu Erwachsenenliteratur als „leicht“ und „problemlos“ eingestuft wird. Dabei ist, wie Katharina Reiß (1982: 1) im Jahre 1982 betont, eine Großzahl von KJL „ausgesprochen schlecht“ übersetzt. Inwiefern diese These tatsächlich zutrifft, ist eine Frage, die bislang nur begrenzt anhand von einzelnen Übersetzungen übersetzungskritisch näher untersucht worden ist (vgl. hierzu z. B. O'Sullivan 2000; Neydim 2003) und darum mit diesem Aufsatz weiter analysiert werden soll. Als Fallbeispiel soll Michael Endes im Jahre 1979 erschienener phantastischer Roman Die unendliche Geschichte und seine Übersetzungen ins Türkische dienen. Der in 40 verschiedene Sprachen übersetzte Fantasyroman ist ein Klassiker der KJL. Die erste türkische Übersetzung des Romans wurde im Jahre 1986 unter dem Titel Bitmeyecek Öykü veröffentlicht. Diese von Saadet Özkal angefertigte Übersetzung erschien im Verlag Ren (Ende 1986). Zehn Jahre später, im Jahre 1996, folgte eine von Saffet Günersel erstellte zweite Übersetzung mit demselben Titel. Diese Übersetzung wurde vom Verlag Kabalcı publiziert (Ende 1996) und unterscheidet sich schon auf den ersten Blick gestalterisch sowie inhaltlich deutlich von der Erstübersetzung Özkals. Eine dritte Übersetzung des Fantasyromans erschien im Jahre 1999 von der Erstübersetzerin Özkal (Ende 1999), erneut unter dem Titel Bitmeyecek Öykü. Bei dem Herausgeber handelt es sich diesmal jedoch um den Verlag Kabalc1, also jenem Verlag, der zuvor die Übersetzung von Günersel veröffentlicht hat. Die letzte und zugleich vierte Übersetzung des Romans erschien im Jahre 2017 im Verlag Pegasus (Ende 2017). Hierbei handelt es sich wiederholt um eine von Özkal erstellte Übersetzung. Insgesamt liegen somit drei Übersetzungen von Özkal vor, die von den drei verschiedenen Verlagen Ren, Kabalcı und Pegasus herausgegeben wurden.

Interessant ist, dass Michael Ende in der Türkei wohl ein bekannter Name ist und eine Reihe seiner Werke in der translationswissenschaftlichen Literatur behandelt worden sind (vgl. z. B. Neydim 2003; Yılmaz 2012; Köprülü 2017), jedoch gibt es, soweit festgestellt werden konnte, bislang keine translationswissenschaftliche Einzeluntersuchung zu den Türkischübersetzungen der Unendlichen Geschichte. Angeregt durch diese Forschungslücke ist die Masterarbeit mit dem Titel Michael Endes Roman Die unendliche Geschichte im Türkischen. Eine übersetzungskritische Analyse (Uğuz 2017) entstanden, auf deren Teilergebnissen die nachstehende Analyse basiert. Thema der Arbeit von Gülsüm Uğuz sind die Übersetzungsstrategien der Übersetzer im Umgang mit „Übersetzungsproblemen“. „Übersetzungsprobleme“ sind, um mit Nord zu sprechen, Aufgaben, die bei der Übersetzung eines AT in einen für eine bestimmte Zielfunktion vorgesehenen ZT gelöst werden müssen (Nord 2011: 120). In der genannten Studie von

1 Nachstehend wird wie folgt abgekürzt: Kinder- und Jugendliteratur: KJL; Ausgangstext: AT, Zieltext: ZT. 
Uğuz werden ausgewählte Übersetzungsprobleme nach einem kindesgerecht und funktional orientierten Analysemodell (Emer O’Sullivan und Christiane Nord) hinsichtlich folgender systematisierter Aspekte analysiert: „Buchtitel“, „Kapiteleinteilung“, „Illustrationen“, „Kapiteleinleitung“, „Kapitelüberschriften“, „Eigennamen“, „,suggestive Atmosphäre der Sprache“ und „assoziative Sprachschwierigkeiten“. Die vorliegende Analyse beschränkt sich angesichts des hier zur Verfügung stehenden Raumes allein auf einen der oben genannten Aspekte - den der „Eigennamen“ -, jedoch wird hierbei die jüngst erschienene vierte Türkischübersetzung des Romans (Ende 2017) ergänzend miteinbezogen.

Wie Uğuz (2017) darlegt, stellen in der Unendlichen Geschichte die Namen von phantastischen Wesen und Orten ein besonderes Übersetzungsproblem dar. Denn die Romanhandlung findet größtenteils in einer grenzenlosen Phantasiewelt statt und der literarische Einfallsreichtum des Autors zeigt sich $u$. a. besonders deutlich bei der Namengebung von im Roman vorkommenden phantastischen Wesen und Orten. Ziel des vorliegenden Aufsatzes ist es übersetzungskritisch zu zeigen, auf welche Übersetzungsverfahren die oben genannten Übersetzer des Romans Die unendliche Geschichte zurückgegriffen haben um das Problem des Übersetzens von Namen von phantastischen Wesen und Orten zu lösen.

Wie Reiß in ihrer Abhandlung „Möglichkeiten und Grenzen der Übersetzungskritik“ (1971) hervorhebt, sind „objektive Kriterien und sachgerechte Kategorien für die Beurteilung von Übersetzungen aller Art” für eine wissenschaftlich fundierte Übersetzungskritik unerlässlich. Diese sollen „das Verlangen nach besseren Übersetzungen“ anregen, was letztendlich dazu führt, dass die „Qualität der Übersetzungen“ steigt (Reiß 1986: 7). In dem vom Reiß vorgelegten übersetzungskritischen Modell geht es hauptsächlich darum „objektiv - d. h. nachprüfbar festzustellen, ob und inwieweit der zu beurteilende Text in der Zielsprache wiedergibt, was der Text in der Ausgangssprache enthielt" (Reiß 1986: 13). Letzterer Anspruch gilt für Übersetzungen von KJL jedoch nur begrenzt, da hier, wie weiter unten ausgeführt wird, ein ziellesergerichtetes Übersetzen als gewichtiger erachtet wird.

Nachstehend werden zunächst die Besonderheiten des kinderliterarischen Übersetzens sowie die translationswissenschaftliche Stellung zur Problematik des Übersetzens von Namen in literarischen Werken kurz skizziert. Sodann wird am Beispiel von Einzelstellen aus den vier Türkischübersetzungen der Frage nachgegangen, wie die beiden Übersetzer mit dem Problem „Übersetzen von Eigennamen“ umgehen. Für die Analyse werden charakteristische Namen aus dem Roman gewählt und nach dem funktionalistischen Textanalysemodell von C. Nord, über das im nachstehenden Abschnitt gesprochen wird, näher betrachtet. Abschließend werden dann die Ergebnisse des Übersetzungsvergleichs nach der funktionalen Übersetzungstheorie von C. Nord unter besonderer Beachtung der von E. O'Sullivan genannten Kriterien für kindeszentriertes Übersetzen bewertet. 


\section{KJL in der Translationswissenschaft}

Der Begriff KJL wird allgemein als Ober- und Sammelbegriff für all jene Werke verwendet, die für Kinder und Jugendliche zwischen 0-25 Jahren produziert werden. Ali Gültekin schlägt eine weitere Differenzierung des Sammelbegriffs KJL vor und unterscheidet dem Alter der anvisierten Lesergruppe folgend zwischen weiteren Subkategorien (Gültekin 2011: 36). Die unendliche Geschichte wird in der Regel für Kinder ab 12 Jahren empfohlen. Der Autor Ende lehnt jedoch eine Klassifizierung in Erwachsenen- und Kinderliteratur ab, denn in seinen Büchern richtet er sich eigentlich nicht nur an Kinder, sondern an das „Kind in uns allen“ (Hocke \& Hocke 2009: 16f.).

Die allgemeine Auffassung der KJL besagt, dass zwei Aspekte bei jeder Art des Übersetzens besonders wichtig sind: die Situation und der Zweck. Nach Riita Oittinen nehmen Übersetzer hierbei Gebrauch von ihrem kulturellen Erbe und ihrer Leseerfahrungen. Bei der Übersetzung von KJL kommt ein dritter besonders wichtiger Aspekt hinzu: das „Bild von Kindern“(Oittinen 2003: 250). Jedoch ist „das Bild von einem Kind“ nicht statisch, sondern wird von mehreren Faktoren, wie Zeit und Kultur mitgeprägt. Mitunter aus den oben genannten Gründen können bei der Übersetzung von KJL Übersetzungsprobleme entstehen. Übersetzungsprobleme in der Erwachsenenliteratur und in der KJL scheinen auf dem ersten Blick dieselben zu sein, doch müssen oft auf andere Lösungswege zurückgegriffen werden.

Bei der Unterscheidung von literarischer Literatur für Erwachsene und KJL wird oftmals die Zielgruppe von Kindern und Jugendlichen als speziell dargestellt und in den Vordergrund gerückt. Nach O'Sullivan muss jedoch vielmehr den charakteristischen Merkmalen der KJL Aufmerksamkeit geschenkt werden als der Adressierung. Die Unterschiede zwischen der KJL und der Allgemeinliteratur werden, so O'Sullivan, vor allem in der „Bestimmung des Gegenstands“, „in der Zugehörigkeit der Kinderliteratur zu zwei Systemen“ und „,in der Asymmetrie der Kommunikation zwischen den an der Handlung Beteiligten" deutlich (O'Sullivan 2000: 111). Oittinen und O'Sullivan haben gemeinsam, dass sie sich auf den kindlichen Leser konzentrieren und dementsprechend ein funktionalistisches Übersetzungskonzept befürworten, welches einen besonderen Wert auf die Rezipientenangemessenheit legt.

Wie oben erwähnt, wird für die vorliegende Studie das funktionalistische Textanalysemodell von Nord herangezogen, da dieses die Forderungen einer kindeszentrierten Übersetzungstheorie, wie sie von Oittinen und O'Sullivan gefordert wird, abdeckt. Nord versteht sich als Funktionalistin und folgt der Skopostheorie von K. Reiß und Hans J. Vermeer (1984), welche den „Translationszweck“ (Skopos) als alleinige „Richtschnur für eine Translation“ sieht. Da nach der Skopostheorie der AT nicht mehr als ein „Informationsangebot“ ist, verliert der AT als Maßstab für die Beurteilung einer Übersetzung seine Bedeutung (Reiß \& Vermeer 1991). Auch Nord erwartet, dass eine Übersetzung die vor dem Übersetzungsprozess festgelegte Funktion in der Zielsituation, die „Funktionsgerechtigkeit“ erfüllt. Jedoch fordert Nord neben der „Funktionsgerechtigkeit“ als oberstes Ziel - im Gegensatz zur Skopostheorie - auch eine 
„bestimmte Form der ,Anbindung““ an den AT und führt den Begriff der „Loyalität“ ein. Translatoren sind, so Nord, gegenüber ihren gesamten Handlungspartnern, also Auftraggeber, ZT-Empfänger und auch AT-Verfasser, zur „Loyalität“ verpflichtet und dürfen diese nicht bewusst täuschen. Sämtliche Abweichungen von dem jeweiligen kulturspezifischen Übersetzungsverständnis müssen den Handlungspartnern mittgeteilt und erklärt werden (Nord 2003: 31ff.). Weiterhin betont Nord, dass aufgrund der tradierten Erwartung von einer literarischen Übersetzung die „Loyalität“ gegenüber dem AT-Autor bewahrt werden muss. Denn: „Was fiktive Charaktere wie zueinander sagen und einander antun, tun sie nicht von sich aus, sondern weil der Autor es so will. Dieses „Wollen“ des Autors oder der Autorin ist der funktionale Angelpunkt, der auch die Übersetzung bestimmt“ (Nord 1997: 38).

Für die ,übersetzungsrelevante Textanalyse“, die nach Nord mit der Analyse des Übersetzungsauftrages beginnen und mit einem Vergleich zwischen AT und ZT fortgeführt werden muss, schlägt Nord die Anlehnung an die sog. Lasswell-Formel vor und unterscheidet zwischen folgenden ,textexternen” und ,textinternen” Faktoren:

„Textexterne Faktoren: Wer (Sender/Textproduzent) übermittelt wem (Adressat), wozu (Intention), über welches Medium (Medium/Kanal), wo (Ort), wann (Zeit), warum (Anlass) einen Text mit welcher Funktion (Textfunktion)?“ (Nord 2003: 24f. und 189ff.)

„Textinterne Faktoren: Worüber (Textthema) sagt er/sie was (Textinhalt), (was nicht) (Präsuppositionen), in welcher Reihenfolge (Textaufbau), unter Einsatz welcher nonverbalen Elemente, in welchen Worten (Lexik), in was für Sätzen (Syntax), in welchem Ton (suprasegmentale Merkmale), mit welcher Wirkung?" (Nord 2003: 24f. und 189ff.)

Für die vorliegende Übersetzungsanalyse ist Nords eigentlich didaktisch gedachte Textanalysemodell insofern bedeutend, als mit Bestimmung der „textexternen“ Faktoren retrospektiv ein Hinweis auf den Skopos der Übersetzungen und die von den Übersetzern gewählte Übersetzungsstrategie erschlossen werden kann. Im Analyseteil wird darum zunächst ein Blick auf die sog. „Paratexte“ geworfen. „Paratexte“ sind nach Genette (2014: 10) alle Texte und Inforationen, die über den ,eigentlichen“ Text hinausgehen und als „Gebrauchsanweisung“ dienen. Dazu mehr weiter unten. Im Anschluss daran werden die „textinternen“ Faktoren anhand repräsentativer Beispiele zu Übersetzungen von Namen von phantastischen Wesen und Orten analysiert.

\section{Analytischer Teil}

\subsection{Eigennamen als Übersetzungsproblem}

Wie in der literaturwissenschaftlichen Forschung bekannt, nehmen Namen in literarischen Werken in der Regel einen wichtigen Platz ein. Die Namengebung von literarischen Figuren ist oft eine Schwierige, da sie eher selten dem Zufall, sondern einer bewussten Entscheidung des Autors unterliegt und mitunter als Mittel zur Rezeptionssteuerung dient. Schwieriger als die Namengebung gilt das Übersetzen von solchen literarischen Namen. Für die 
Translationswissenschaft sind Namen aus Märchen, Kinderbüchern und literarischen Texten von besonderem Interesse, vor allem wenn sie z.B. einen konnotativen Wert haben und dadurch ein Übersetzungsproblem darstellen. Zu den Aufgaben eines Übersetzers gehört es nach Andreas F. Kelletat im Werk vorkommende Namen, die einen konnotativen Wert haben, zu erkennen, um diese dann nicht nur in eine neue Sprache, sondern auch zugleich in eine neue Kultur zu übersetzen (Kelletat 2003: 297f.).

Auch im Roman Die unendliche Geschichte spielen Namen eine besondere Rolle. Da sich die Romanhandlung zum größten Teil in einer Phantasiewelt zuträgt, wird der literarische Einfallsreichtum von Ende besonders bei der Namengebung deutlich. Dies bestätigt Ende selbst indem er die Namengebung in literarischen Werken als einen ,schöpferischen Akt“ beschreibt, indem eine Bindung zu jemanden oder etwas aufgebaut und ihm Wirklichkeit verleiht wird (Hocke \& Hocke 2009: 146). Der Leser wird mit zahlreichen Neubildungen von Eigennamen konfrontiert, welche die Einmaligkeit und Fremdheit von Phantásien, die phantastische Welt in der Unendlichen Geschichte, unterstreichen. Diese sind für den kindlichen Leser erst dann verständlich, wenn sie vom Autor durch metasprachliche Kommentare charakterisiert werden. Die Charaktere „Bastian Balthasar Bux“ und „Karl Konrad Koreander“ gehören der realen Welt in Die unendliche Geschichte an und sind wohlmöglich die einzigen geläufigen Namen, die im gesamten Roman vorkommen. Alle anderen Namen von Geschöpfen oder Orten in Phantásien sind Produkte des literarischen Einfallsreichtums Endes (Aschenberg 1991: 90f.). Diese stellen für die Übersetzer, wie unten an verschiedenen Beispielen gezeigt werden soll, ein Übersetzungsproblem dar.

Hierbei ist es interessant der Frage nachzugehen, welcher Übersetzungsstrategie die Übersetzer Özkal und Günersel gefolgt sind und mit welchen „Übersetzungsverfahren“ sie das besagte Übersetzungsproblem versucht haben zu lösen. Bei „Übersetzungsverfahren“ handelt es sich um „Techniken der Übersetzung“ die auf Textabschnitte angewandt werden und von der Übersetzungsmethode und vom Sprachen- bzw. Kulturpaar abhängen. Zu den bekanntesten Übersetzungsverfahren gehören Entlehnung, Ersetzung, Expansion, Reduktion, Modulation, Adaption u. a. (Schreiber 2003: 151f.; Berk 2005: 104). In der translationswissenschaftlichen Forschung wird die Wiedergabe von Namen als ein Problem gesehen, für das es keine allgemeingültige „Lösung“ gibt. Es können - je nach Textsorte und Forderung der Übersetzung verschiedene Übersetzungsstrategien und Übersetzungsverfahren angewandt werden (Kelletat 1999; Newmark 2004).

Wie oben erwähnt, sind „Paratexte“ für übersetzungskritische Analysen von großer Bedeutung, da es durch sie möglich ist Hinweise zum Übersetzungsauftrag einer Übersetzung und zur Übersetzungsstrategie des Übersetzers zu erschließen. Zu den „Paratexten“ einer Übersetzung wird nach Genette (2014: 10) die gesamte Aufmachung zur Übersetzung gezählt, welche Buchumschlag, Klappentext, Vor- und Nachworte des Übersetzers oder Herausgebers sowie Interviews, Berichte des Übersetzers beinhaltet. Ein Blick auf die „Paratexte“ der hier 
zu untersuchenden vier Übersetzungen zeigt, dass kein Hinweis auf den Skopos der jeweiligen Übersetzung und die vom Übersetzer gewählte Übersetzungsstrategie vorliegt. Hieraus folgend kann davon ausgegangen werden, dass den Romanübersetzungen als Translationszweck keine Funktionsänderung, z. B. in Form einer „Bearbeitung“, zugrunde liegt.

\subsection{Beispielanalysen}

In Endes Roman Die unendliche Geschichte begegnen sich eines Tages die vier verschiedenen phantastischen Geschöpfe „Das Irrlicht“, „Der Winzling“, „Der Nachtalb“ und „Der Felsenbeißer“ im Haulewald. In kurzer Zeit bemerken sie, dass alle als Boten unterwegs sind, um über ein Ereignis zu berichten, dass sich in ganz Phantásien verbreitet. Jene vier Geschöpfe heißen der Reihe nach „Blubb“, „Ückück“, „Wúschwusul“ und „Pjörnrachzarck“.

\begin{tabular}{|l|l|l|l|l|}
\hline \multicolumn{5}{|l|}{ Beispiel 1 } \\
\hline AT: & Blubb (S. 22) & Ückück (S. 22) & Wúschwusul (S. 22) & Pjörnrachzarck (S. 22) \\
\hline ZT 1: & Blubb (S. 24) & Ückück (S. 24) & Wuschwusul (S. 24) & Pjörnrachzarck (S. 24) \\
\hline ZT 2: & Blubb (S. 27) & Ückück (S. 27) & Wuschwusul (S. 27) & Pjörnrachzarck (S. 27) \\
\hline ZT 3: & Blubb (S. 27) & Ückück (S. 27) & Wuschwusul (S. 27) & Pjörnrachzarck (S. 27) \\
\hline ZT 4: & Blubb (S. 27) & Ückück (S. 27) & Wuschwusul (S. 27) & Pjörnrachzarck (S. 27) \\
\hline
\end{tabular}

Wie die obige Tabelle zeigt, wurden die Namen der vier Geschöpfe in allen Türkischübersetzungen direkt aus dem AT übernommen. Die Namen mögen auf dem Blick seltsam zu erscheinen, doch handelt es sich keines Falles um eine willkürliche Namengebung. Ende unterstützt seine Namengebung mit Hinweisen auf ihre Sprechweise und hilft dem ZT-leser dadurch sich den jeweiligen Charakter des Geschöpfes vorstellen zu können. Es handelt sich hierbei also um eine onomatopoetische Namengebung, welche klangsymbolische Namen verwendet um einen Charakter durch seinen Namen vorzustellen. Wie unten an verschiedenen Beispielen weiter ausgeführt wird, haben auch diese Namen erst mit den im Roman beschriebenen Erklärungen und Kommentaren einen Sinn. So besteht der Name „Ückück“ aus zwei aufeinanderfolgenden gleichen Silben um beginnt mit dem Umlaut „̈̈“. Bei der Artikulierung des Umlauts werden die Lippen spitz geformt, womit die piepsige Stimme des Winzlinges betont werden soll (Kromp 2008: 89): „Der Winzling stand auf, ging ein paar Schrittchen auf den Ankömmling zu und piepste: ,Wenn ich richtig sehe, so sind auch Sie in Ihrer Eigenschaft als Bote hier?““ (Ende 1979: 22). Wie im obigen Zitat zu erkennen, beschreibt Ende selbst die Sprechweise des Winzlings näher indem er ,piepste“ anstatt von „,sagte“ verwendet. Das äußere Aussehen des Geschöpfes wird im Roman passend dazu als ein ,überaus feingliedriges Kerlchen in einem bunten Anzüglein und mit einem roten Zylinder auf dem Kopf" beschrieben, dass leicht zu übersehen ist (Ende 1979: 20). Grafisch wird dies zusätzlich durch die im Roman abgebildeten Illustrationen von Roswitha Quadflieg unterstützt (Ende 1979: 18). 
Auch der Name des Felsenbeißers „Pjörnrachzarck“ bedient sich der Klangsymbolik. Die „knatternde“ Sprechweise des Geschöpfes, die dem Zermalmen von Steinen gleicht, wird durch den Klang des Namens nachgeahmt: „,Angenehm!‘, knarrte der Felsenbeißer, ,ich bin Pjörnrachzarck““ (Ende 1979: 22). Im Roman wird der Felsenbeißer passend zu seinem Namen als ein zehn Fuß langer Riese aus grauem Stein beschrieben der mit einem aus Gestein angefertigten Fahrrad unterwegs ist auf einem Fahrrad mit Rädern aus Stein dargestellt (Ende 1979: 19f.).

Der Nachtalb „Wúschwusul“ wird als ein pechschwarzes einer fellbedeckten Raupe ähnelndes Geschöpf mit schwarzen Wuschelhaaren und großen Kugelaugen dargestellt, das mit einer Fledermaus unterwegs ist (Ende 1979: 20). Die wiederholende Verwendung des Vokals „u“ im Namen und Ausrufe wie z.B. „Huhu!“” (Ende 1979: 22, 24 u. 25) und „Huuu!“” (Ende 1979: 23) machen auf die typische Artikulation des Nachtalbs aufmerksam. Der Name verweist außerdem auf die Wuschelhaare, die ein wichtiges Merkmal des Nachtalbs sind:

„Die zweite Gestalt, die rechts vom Feuer saß, war ein Nachtalb. [...] Er gestikulierte heftig beim Sprechen mit zwei winzigen rosa Händchen und dort, wo unter den schwarzen Wuschelhaaren vermutlich das Gesicht war, glühten zwei große kreisrunde Augen wie Monde“ (Ende 1979: 20).

Die direkte Übernahme des Namen „Wúschwusul“ in den Türkischübersetzungen führt dazu, dass der Verweis auf die Wuschelhaare durch die Klangsymbolik selbst ausfällt. Der Leser erfährt sowohl aus dem Kontext als auch durch die grafischen Darstellungen im Roman, dass der Nachtalb „Wúschwusul“"Wuschelhaare hat, doch ein literarisches Element des Autors geht durch die Übernahme verloren. O’Sullivan (2000: 207f.) bezeichnet einen Verlust dieser Art als ,Zähmung sprachlicher Kreativität“.

Wie in der obigen Tabelle zu sehen ist, wird auch die Schreibweise aller vier Namen direkt übernommen. Die Wirkung jener klangsymbolischen Namen ist somit nicht mehr gesichert. Der ZT-Leser wird mit Buchstabenkombinationen, wie z.B. „,ck“, „,ch“ und „sch“ konfrontiert, die im Türkischen nicht vorhanden sind und dessen Aussprache dem ZT-Leser nicht bekannt sein dürfte. Auch bei der Aussprache von einzelnen Buchstaben wie z.B. bei „c“ und ,j“ gibt es Differenzen zwischen dem Deutschen und Türkischen. Diese Unterschiede hindern den ZT-Leser davor eine Verbindung zwischen der Klangsymbolik der Namen und den Erklärungen und Kommentaren zu den Geschöpfen herzustellen. Das oben geschilderte Problem könnte durch die Anpassung von Buchstaben und Buchstabenkombinationen der AS an das System der ZS gelöst werden. Die Namen „Ückück“ und „Wúschwusul“ könnten nach einer orthographischen Assimilation ans Türkische z.B. „Ükük“ und „Vuşvuzul“ lauten. 


\begin{tabular}{|l|c|}
\hline Beispiel 2 \\
\hline AT: & Die Uralte Morla (S. 58, 59) oder Morla (S. 58, 59, 60) \\
\hline ZT 1: & Kadim Morla (S. 65, 66, ) oder Morla (S. 65, 66, 67) \\
\hline ZT 2: & Asrrlık İhtiyar Morla (S. 70, 71) oder Morla (S. 70, 71, 72) \\
\hline ZT 3: & Kadim Morla (S. 72, 74 ) oder Morla (S. 72, 73, 74) \\
\hline ZT 4: & Kadim Morla (S. 72, 73, 74) oder Morla (S. 72, 73, 74) \\
\hline
\end{tabular}

„Die uralte Morla“ ist eine alte, weise, riesige Schildkröte im Roman Die unendliche Geschichte. Sie ist so groß, dass „Hornberg“, ein Ort Phantásiens, das tief in den „Sümpfen der Traurigkeit“" liegt, eigentlich ein Teil von ihr ist. Der Name des phantastischen Geschöpfes besteht aus dem Namen „Morla“ und dem vorgestellten Adjektiv „uralt““. Der Name „Morla“ wird in allen vier Türkischübersetzungen direkt übernommen. Wie oben zu sehen ist, wird jedoch das vorgestellte Adjektiv „uralt“ im Türkischen durch „Kadim“ oder „Asırlık İhtiyar“ ersetzt. Diese teils Übernahme, teils Übersetzung hat zur Folge, dass das im AT vorhandene Wortspiel zwischen dem Namen „Morla“ und dem Ort, wo sie lebt, verloren geht. Denn „Morla“ lebt in einem Sumpfgebiet: „Weit, weit von hier im Norden liegen die Sümpfe der Traurigkeit. Mitten in diesen Sümpfen ragt der Hornberg auf. Dort wohnt die Uralte Morla“ (Ende 1979: 54). Im Deutschen sind die Begriffe „Sumpf“ und „Moor“ synonym zueinander. Ende macht sich Gebrauch von dieser Bedeutungsgleichheit und spielt mit dem Namen „Morla“ womöglich auf „Moor“ an, da die „Uralte Morla“ im Sumpf- bzw. Moorgebiet lebt. Das hier verwendete sprachstilistisch-literarische Element geht in den Türkischübersetzungen verloren, da das im AT verwendete Wortspiel in der ZS in dieser Art nicht wiedergegeben werden kann. Hier werden die Übersetzer von Die unendliche Geschichte mit einem in der Translationswissenschaft bekannten Übersetzungsproblem konfrontiert, denn:

„Jede Sprache verbindet Form und Bedeutung auf weitgehend arbiträre und sprachspezifische Weise, so daß die Verknüpfung von formaler Ähnlichkeit und semantischer Verschiedenheit, die ein Wortspiel in einer Sprache möglich macht, in einer anderen Sprache sehr oft nicht wiedergegeben werden kann“ (Delabastita 2003: 285).

\begin{tabular}{|c|c|c|c|c|}
\hline \multicolumn{5}{|c|}{ Beispiel 3} \\
\hline AT: & Lirr (S. 125) & Baureo (S. 126) & Schirk (S. 126) & Mayestril (S. 126) \\
\hline ZT 1: & Lirr (S. 134) & Baureo (S. 134) & Schirk (S. 134) & Mayestril (S. 134) \\
\hline ZT 2: & Lirr (S. 144) & Baureo (S. 144) & Schirk (S. 144) & Mayestril (S. 144) \\
\hline ZT 3: & Lirr (S. 153) & Baureo (S. 153) & Schirk (S. 153) & Mayestril (S. 153) \\
\hline ZT 4: & Lirr (S. 149) & Baureo (S. 149) & Schirk (S. 149) & Mayestril (S. 149) \\
\hline
\end{tabular}


Wie die obige Tabelle zeigt, wurden die Namen der vier Windriesen „Lirr“, „Baureo“, „Schirk“ und „Mayestril“" in allen Türkischübersetzungen so wie sie sind beibehalten. Besonders bei drei von ihnen ist eine Ähnlichkeit zu Windenamen, die in Wirklichkeit existieren, erkennbar. Es wird daher vermutet, dass die Namen der Windriesen „Baureo“, „Schirk“ und „Mayestril““ von den Windenamen „Boreas“, „Schirocco“ und „Mistral““ abgewandelt wurden (http:// ggweather.com/winds.html). Die Namengebung des im Roman aus westlicher Richtung wehenden Windes „Mayestril“ soll an dieser Stelle etwas näher betrachtet werden. Auf dem ersten Blick stößt die Ähnlichkeit zum Begriff „Majestät“ ins Auge. Es handelt sich hierbei möglicherweise um eine bewusste Entscheidung Endes, da „Mistral“ ursprünglich ein starker Fallwind aus nordwestlicher Richtung ist. Diese Stärke wird durch die die Ähnlichkeit von „Mayestril““ zum Begriff „Majestät“ betont, da dieser mit Erhabenheit, Größe, Würde und Hoheit in Verbindung gebracht wird (Duden online, 2019, Stichwort: „Majestät“).

Darüber hinaus ist folgendes festzustellen: Die Bezeichnung ,mistral“ stammt ursprünglich von „maestre“ (frz. maître) ab und bedeutet Hauptwind. Somit wird die Stärke und Relevanz des Windes „Mayestril“ auch semantisch betont. Ende betont diese Stärke vermutlich durch die Verwendung des Namens „Mayestril““, indem er auf die Anrede „Majestät““ anspielt (Mládková 2009: 59).

Die türkische Entsprechung des Begriffs „Majestät“ lautet „,majeste“. Der Begriff wurde durch eine orthographisch assimilierte Entlehnung aus dem Französischen in den türkischen Wortschatz aufgenommen. Beide Begriffe ähneln sich phonologisch stark, sodass das weiter oben erläuterte sprachstilistisch-literarische Element des Autors als Konnotation in den Türkischübersetzungen erhalten wurde. Diese Konnotation hätte jedoch durch eine Buchstabenadaption ans Türkische verstärkt werden können, indem das „Y“ in „Mayestril““ durch ein ,J“ ersetzt wird.

\begin{tabular}{|l|c|}
\hline \multicolumn{2}{|l|}{ Beispiel 4 } \\
\hline AT: & Der Haulewald (S. 19, 20) \\
\hline ZT 1: & Aşka Çağrı Ormanı (S. 21) \\
\hline ZT 2: & Cinler Ormanı (S. 23) \\
\hline ZT 3: & Aşka Çağrı Ormanı (S. 21) \\
\hline ZT 4: & Amansı Orman (S. 23) \\
\hline
\end{tabular}

„Der Haulewald“ ist ein wichtiger Ort in Die unendliche Geschichte, da sich die vier Boten „das Irrlicht“, „der Winzling“, „,der Nachtalb“ und „der Felsenbeißer“ erstmals hier begegnen und bemerken, dass alle unterwegs sind um der „Kindlichen Kaiserin“ vom „Nichts“ zu berichten. Der Ort wird als ein großer, düsterer Wald Phantásiens beschrieben, wo das Heulen des Windes zwischen den Zweigen ertönt (Hocke \& Hocke 2009: 109). Wie oben zu sehen ist, wird der „Haulewald“ in der ersten und zweiten Übersetzung (ZT 1 und ZT 3) von Özkal mit 
„Aşka Çağrı Ormanı““ [Der Wald Aufruf zur Liebe] ins Türkische übersetzt. Hierzu verwendet sie das Übersetzungsverfahren der Modulation, sodass der erste Satz des ersten Kapitels mit dem Buchstaben „A“ eingeleitet wird. Denn die besondere Aufmachung des Romans erfordert, dass jedes der 26 Kapitel mit einem Buchstaben in der alphabetischen Reihenfolge von A bis $\mathrm{Z}$ beginnt. Dennoch ist Özkals Entscheidung für „Aşka Çağrı Ormanı“ semantisch schwer nachvollziehbar, denn wie oben bereits beschrieben handelt es sich bei dem „Haulewald“ um einen großen Wald mit einer düsteren Atmosphäre. Durch die Übersetzung mit „Aşka Çağr1 Ormanı" wird dagegen eine positiv einladende Stimmung, also genau das Gegenteil, erweckt. Günersel (ZT 2) löst das Problem hinsichtlich der Einleitung mit dem Buchstaben „A“ durch die Hinzufügung eines in Türkisch mit „A“ beginnenden Wortes und übersetzt „Haulewald“ mit „Cinler Ormanı“ [Dschinnwald]. Im islamischen Volksglauben wird unter dem Begriff „cin“ [Dschinn] „,böser Geist“ verstanden (Duden online, 2019, Stichwort: „Dschinn“). Mit dieser Übersetzung schafft es Günersel die düstere Stimmung des „Haulewalds“ im Vergleich zu „Aşka Çağrı Ormanı“ eher wiederzugeben. Günersels Übersetzung gilt somit sowohl funktionsgerecht als auch „loyal“ im Sinne von Nord. In der Viertübersetzung des Romans wählt Özkal die Bezeichnung „Amansız Orman“ [gnadenloser/bösartiger Wald], womit auch sie es schafft eine „funktionsgerechte“ und „loyale“ Übersetzung zu erzielen.

\begin{tabular}{|l|c|}
\hline Beispiel 5 & \\
\hline AT: & Der Magnolienpavillon (S. 30, 33) \\
\hline ZT 1: & Manolya Pavyonu (S. 33, 37) \\
\hline ZT 2: & Manolya Pavyonu (S. 36, 41) \\
\hline ZT 3: & Manolya Köşkü (S. 37, 41) \\
\hline ZT 4: & Manolya Köşkü (S. 36f., 41) \\
\hline
\end{tabular}

An der höchsten Stelle und im Herzen des „Elfenbeinturms“ befindet sich der „Magnolienpavillon“. Der „Elfenbeinturm“ ist der Wohnort der „Kindlichen Kaiserin“ und unterscheidet sich sehr stark von dem uns bekannten Bild eines Turmes. Er sieht von weitem wie ein spitzer Bergkegel aus, der wie eine Schneckenmuschel in sich gedreht ist. Der „Magnolienpavillon“ selbst gleicht einer weißen Magnolienknospe und öffnet sich bei schönen Vollmondnächten (Hocke \& Hocke 2009: 70ff.). Da durch diese Beschreibung offensichtlich ist wie der „Magnolienpavillon“ in Die unendliche Geschichte aussieht, passt folgende Definition des Begriffes „Pavillon“ am geeignetsten: „,zu einem größeren Komplex gehörender selbstständiger Bau. Der „Magnolienpavillon“ wird sowohl in der Erstübersetzung von Özkal als auch in der Zweitübersetzung von Günersel mit „Manolya Pavyonu“ ins Türkische übersetzt. Da der Begriff „Pavyon“ im Türkischen auch als Bezeichnung für einen Nachtclub verwendet wird, besteht die Gefahr das die Übersetzung als „Manolya Pavyonu“ bei dem kindlichen Leser zu Missverständnissen führt (Türk Dili Kurumu, TDK, online, 
2019, Stichwort: „pavyon“). In der dritten und vierten Übersetzung des Romans hebt Özkal eventuelle Missverständnisse auf, indem sie anstatt von „Manolya Pavyonu“ die Bezeichnung „Manolya Köşkü“، verwendet.

\begin{tabular}{|l|c|}
\hline \multicolumn{2}{|l|}{ Beispiel 6 } \\
\hline AT: & Das gräserne Meer (S. 38) \\
\hline ZT 1: & Ot Denizi (S. 43) \\
\hline ZT 2: & Yeşeren Deniz (S. 47) \\
\hline ZT 3: & Ot Denizi (S. 48) \\
\hline ZT 4: & Ot Denizi (S. 47) \\
\hline
\end{tabular}

Die Heimat der Grasleute namens „Das gräserne Meer“ ist eine Prärie in Phantásien. Der Ort wurde so benannt, da er so groß und flach wie ein Meer ist und sehr hohes, saftiges Gras in der Prärie wächst. An windigen Tagen weht der Wind über das hohe Gras, wodurch Wellen über die Ebene ziehen und es wie Wasser rauscht (Ende 1979: 38). Wie die obige Tabelle zeigt, entscheidet sich Özkal das ,gräserne Meer“ in allen drei Türkischübersetzungen (ZT 1, ZT 3 und ZT 4) mit „Ot Denizi“ zu übersetzen. Im Türkischen wird das Adjektiv „ot“ jedoch eher als Entsprechung für Unkraut verwendet. Der Begriff „Gras“" wird hingegen mit „,̧̧ayır“ und „,̧imen“ ins Türkische übersetzt. Die Verwendung von „ot“ bringt die Gefahr mit sich, dass der ZT-Leser einen negativen Eindruck von der Prärie bekommt. Ende beschreibt den Ort jedoch ganz im Gegenteil als ,[...] eine Prärie, die tatsächlich soweit und groß und flach war wie ein Meer [ist]. Saftiges Gras wuchs mannshoch, und wenn der Wind darüber hinstrich, zogen Wellen über die Ebene wie auf dem Ozean und es rauschte wie Wasser“ (Ende 1979: 38). In der Zweitübersetzung von Günersel wird das „Das gräserne Meer“ mit „Yeşeren Deniz“ in Türkische übersetzt. Die Infinitivform des hier verwendeten Begriffs ,yeşeren“ ist ,yeşermek“ und bedeutet im Deutschen ,grün werden“. Die Bezeichnung „Yeşeren Deniz“ kann somit mit „das grün werdende Meer“ ins Deutsche übersetzt werden. Diese Entscheidung von Günersel ist semantisch nachvollziehbar, da Gras mit der Farbe Grün assoziiert wird. Somit passen die Benennung des Ortes und die oben von Die unendliche Geschichte zitierte Beschreibung des Ortes zusammen. Der ZT-Leser assoziiert somit ein ähnliches Bild vom ,gräsernen Meer“ wie der AT-Leser, womit die Übersetzung sowohl „,funktionsgerecht“, als auch „loyal““ wird.

\section{Fazit}

Im Rahmen dieser Arbeit sollte übersetzungskritisch gezeigt werden, auf welche Übersetzungsverfahren die zwei Übersetzer Saadet Özkal (= ZT 1, ZT 3 und ZT 4) und Saffet Günersel (=ZT 2) zurückgegriffen haben, um das Problem des Übersetzens von Namen von phantastischen Wesen und Orten in Die unendliche Geschichte zu lösen. Wie die obigen Beispielanalysen zeigen, liegen sprachliche Differenzen zwischen ZT 1 und ZT 2 vor, doch im 
Allgemeinen schaffen es beide Türkischübersetzungen „Funktionsgerechtigkeit“" und „Loyalität“ zu erzielen. Zusammenfassend lässt sich sagen, dass somit dem ZT-Leser ebenso wie dem ATLeser ein unterhaltsamer, aber gleichzeitig auch sehr anspruchsvoller Roman geboten wird.

Die oben dargestellten Ergebnisse zu ZT 1 rechtfertigen die Aussage, dass Özkals Übersetzung etwas ,verfremdend“ wirkt, da sie sich bei der Übersetzung von Namen teilweise streng an die Formulierungen des AT orientiert. Viele oben diskutierte Beispiele zeigen jedoch, dass Özkal sich methodisch an eine Rezipienten berücksichtigende „funktionsgerechte“ Übersetzung anlehnt. Wie der Übersetzungsvergleich darstellt, erfordert dies „Adaptionen“ verschiedener Art. Diese haben wiederum oft zur Konsequenz, dass sprachstilistische Mittel des Erzählers verloren gehen. „Die Zähmung sprachlicher Kreativität“ kommt, um mit O’Sullivan zu sprechen, bei ZT 1 somit oftmals zum Vorschein.

Durch die Auswertung der Ergebnisse zu Günersels Übersetzung (=ZT 2), konnte nachgewiesen werden, dass diese im Vergleich zu ZT 1 einen größeren Wert auf eine rezipientengerichtete Übersetzung legt. Aufgrund der Unterschiede des Sprachenpaars wird jedoch auch Günersel mit Problemen konfrontiert, die auch von ihm nur bis zu einem gewissen Maße gelöst werden können, so wie z. B. das Übertragen von Sprachspielen. Zusammenfassend lässt sich sagen, dass Günersel eine „,funktionsgerechte“ Übersetzung anstrebt, die aufgrund verschiedener Anpassungen an die ZK als kindeszentriert bewertet werden kann. Doch „Adaptionen“ haben zur Konsequenz, dass charakteristische Kennzeichen des Werkes verloren gehen.

Anhand von den Ergebnissen zu Saadet Özkals Zweitübersetzung (= ZT 3) konnte nachgewiesen werden, dass es sich bei dieser um eine überarbeitete Neuveröffentlichung ihrer Erstübersetzung handelt. Es wurden lediglich kleine Verbesserungen vorgenommen und veraltete Formulierungen unter Beachtung des kindlichen Lesers zeitgemäß angepasst. Dasselbe trifft auch für die Saadet Özkals Drittübersetzung (= ZT 4) zu. Auch hier werden lediglich minimale Verbesserungen vorgenommen und zeitgemäß angepasst. Interessant wäre es im Anschluss an die vorliegende Studie der Frage nachzugehen, welche weiteren Neuerungen in der letzten Neuübersetzung von Özkal vorgenommen wurden.

Peer-review: Externally peer-reviewed.

Conflict of Interest: The authors have no conflict of interest to declare.

Grant Support: The authors declared that this study has received no financial support. 


\section{LITERATURVERZEICHNIS/REFERENCES}

Aschenberg, H. (1991). Eigennamen im Kinderbuch: eine textlinguistische Studie, Tübingen: Gunter Narr.

Berk, Ö. (2005). Kuramlar Işı̆̆ı̆nda Açıklamalı Çeviribilim Terimcesi, İstanbul: Multilingual.

Delabastita, D. (2003). 'Wortspiele' (Übers.: P. Kußmaul) in M. Snell-Hornby, H. G. Hönig, P. A. Kußmaul \& P. A. Schmitt (Hrsg.), Handbuch Translation (2. verb. Aufl.). Tübingen: Stauffenberg, 285-288.

Ende, M. (1979). Die unendliche Geschichte, Stuttgart: Thienemann.

Ende, M. (1986). Bitmeyecek Öykü (Übers.: S. Özkal), Istanbul: Ren.

Ende, M. (1996). Bitmeyecek Öykü (Übers.: S. Günersel), Istanbul: Kabalc1.

Ende, M. (1999). Bitmeyecek Öykü (Übers.: S. Özkal), Istanbul: Kabalc1.

Ende, M. (2017). Bitmeyecek Öykü (Übers.: S. Özkal), Istanbul: Pegasus.

Genette, G. (2014). Paratexte. Das Buch vom Beiwerk des Buches. Mit einem Vorwort von Harald Weinrich (Übers.: D. Hornig), (5. Aufl.), Frankfurt: Suhrkamp.

Gültekin, A. (2011). Çocuk ve Gençlik Edebiyatı Yazıları. İstanbul: Erdem.

Hocke, P., \& Hocke, R. (2009. Michael Ende. Die unendliche Geschichte. Das Phantásien-Lexikon. Stuttgart/ Wien: Thienemann.

Kelletat, A. F. (2003). 'Eigennamen' in M. Snell-Hornby, H. G., P. A. Kußmaul \& P. A. Schmitt (Hrsg.), Handbuch Translation (2. verb. Aufl.). Tübingen: Stauffenberg, 297-298.

Köprülü, S. G. (2017). 'Übersetzungsstrategien der Kinderliteratur. Eine Analyse am Beispiel der türkischen Übersetzung von Michael Endes Der Teddy und die Tiere', Diyalog Interkulturelle Zeitschrift Für Germanistik, 5 (2), 182-194. (online) http://dergipark.gov.tr/diyalog/issue/34459/379322, [16. Mai 2019].

Koller, W. (2011). Einführung in die Übersetzungswissenschaft (6. Aufl.), Wiebelsheim: Quelle und Meyer.

Kromp, I. (2008). Eigennamen in der deutschen und polnischen Kinderliteratur unter textlinguistischem und translatorischem Aspekt (Bd. 24), Frankfurt am Main: Peter Lang.

Mládková, T. (2009) Michael Endes Unendliche Geschichte und ihre Übersetzungen ins Tschechische und Norwegische. Eine Übersetzungskritik. Thesis (MA). Norwegen: Universitas Osloensis). (online) https:// www.duo.uio.no/bitstream/handle/10852/25654/ Masteroppgave.ferdig.pdf?sequence=1 [15. Mai 2019].

Newmark, P. (2004). 'Names as a translation problem' in H. Kittel, A. P. Frank, N. Greiner, T. Hermans, W. Koller, J. Lambert, ... J. Lambert (Hrsg.), Übersetzung: Ein internationales Handbuch zur Übersetzungsforschung (Handbücher zur Sprach- und Kommunikationswissenschaft; 26.1) (Bd. 26.1). Berlin / New York: Walter de Gruyter, 527-530.

Neydim, N. (2003). 80 Sonrası Paradigma Değişimi Açısından Çeviri Çocuk Edebiyatı, İstanbul: Bu Yayınevi.

Nord, C. (1997). 'So treu wie möglich? Die linguistische Markierung kommunikativer Funktionen und ihre Bedeutung für die Übersetzung literarischer Texte' in R. Keller (Hrsg.), Linguistik und Literaturübersetzen. Tübingen: G. Narr, 35-59.

Nord, C. (2003). Textanalyse und Übersetzen. Theoretische Grundlagen, Methoden und didaktische Anwendung einer übersetzungsrelevanten Textanalyse (3. Aufl.), Tübingen: Julius Groos.

Nord, C. (2011). Funktionsgerechtigkeit und Loyalität. Theorie, Methode und Didaktik des funktionalen Übersetzens, Berlin: Frank \& Timme, 115-125.

O’Sullivan, E. (1991/1992). ‘Kinderliterarisches Übersetzen', Fundevogel, 93/94 Dezember/Januar, 4-9. 
Namen von phantastischen Wesen und Orten in den Türkischübersetzungen von Michael Endes...

O’Sullivan, E. (2000) Kinderliterarische Komparatistik, Heidelberg: Universitätsverlag C. Winter.

Oittinen, R. (2003). 'Kinderliteratur' (Übers.: P. Kußmaul) in M. Snell-Hornby, H. G., P. A. Kußmaul \& P. A. Schmitt (Hrsg.), Handbuch Translation (2. verb. Aufl.). Tübingen: Stauffenberg, 250-253.

Pedrini, E. (2014) Die Übersetzung von Bilderbüchern: Das Verhältnis zwischen Text und Bild, Hamburg: Diplomica Verlag.

Reiss, K. (1982) 'Zur Übersetzung von Kinder- und Jugendbüchern. Theorie und Praxis', Lebende Sprachen, 27 (1), 7-13.

Reiß, K. (1986). Möglichkeiten und Grenzen der Übersetzungskritik: Kategorien und Kriterien für eine sachgerechte Beurteilung von Übersetzungen (3. Aufl.), München: Hueber.

Reiß, K., \& Vermeer, H. J. (1991). Grundlegung einer allgemeinen Translationstheorie (2. Aufl.), Tübingen: M. Niemeyer.

Schreiber, M. (2003). 'Übersetzungstypen und Übersetzungsverfahren’ in M. Snell-Hornby, H. G., P. A. Kußmaul \& P. A. Schmitt (Hrsg.), Handbuch Translation (2. verb. Aufl.). Tübingen: Stauffenberg, 151-154.

Stolze, R. (2001). Übersetzungstheorien. Eine Einführung (3., aktual. Aufl.), Tübingen: Gunter Narr Verlag.

Uğuz, G. (2017). Michael Endes Roman 'Die unendliche Geschichte' im Türkischen. Eine übersetzungskritische Analyse. Thesis (MA). Eskişehir: Anadolu Üniversitesi. (online) https://tez.yok.gov.tr/UlusalTezMerkezi/ TezGoster?key=RrI-Krk3A-RkF4YfHofuk-C5 WaKOhkp4VGfgBYyDjftg3y4rD-a5kvkOy_Z2viLv [10. Mai 2019].

Yılmaz, T. (2012). Michael Ende 'nin 'Momo’'Adlı Eserinin Türkçe Çevirisine Eleştirel Yaklaşım. Thesis (MA). Ankara: Gazi Üniversitesi. (online) https://tez.yok.gov.tr/UlusalTezMerkezi/TezGoster?key=RYan9_SZ7Eir3xdWGXBiA MpX0rT-pMnnMr58e0kdNVHfImuCTiNveXkVqhZFZ2a [11. Mai 2019]. 